The ASTROPHysical Journal SUPPLEMENT SERIES, 90:623-630, 1994 February

(C) 1994. The American Astronomical Society. All rights reserved. Printed in U.S.A.

\title{
TURBULENT ACCELERATION IN SOLAR FLARES
}

\author{
D. B. MELROSE ${ }^{1}$ \\ Research Centre for Theoretical Astrophysics, University of Sydney, NSW 2006 Australia \\ Received 1993 January 25; accepted 1993 May 13
}

\begin{abstract}
Turbulent acceleration in the impulsive phase of solar flares is reviewed, with the emphasis on bulk energization of nonrelativistic electrons and prompt acceleration of the gamma-ray emitting nonrelativistic ions and relativistic electrons. The primary energy release in a flare cannot be due to collisional dissipation. Anomalous resistivity requires that the current flows in many narrow channels with the current density above threshold for current instability. The bulk energization of the electrons is due to the damping of low-frequency electrostatic turbulence generated by the current instability. This turbulence also limits the rate a nonthermal electron tail forms due to runaway acceleration. Stochastic and gyroresonant acceleration by MHD turbulence are discussed briefly, emphasizing the need for preacceleration. Stochastic acceleration is favorable for the gamma-ray emitting particles only if an adequated source of the MHD turbulence can be identified.
\end{abstract}

Subject headings: acceleration of particles - MHD — Sun: flares — turbulence

\section{INTRODUCTION}

In the impulsive phase of solar flares there is copious acceleration of electrons to deca-keV energies on a time scale $\sim 100$ ms (e.g., Ramaty et al. 1980) and associated acceleration of ions to deca-MeV energies of a time scale $\sim 1 \mathrm{~s}$ (e.g., Hudson 1985 ). The acceleration of the electrons is sometimes referred to as bulk energization, because it seems that effectively all the electrons ( $u p$ to $10^{39}$ ) in a portion of the flaring region have their energy increased manyfold. These electrons are detected through their hard X-ray emission: the observed hard X-ray bursts require precipitation of $\gtrsim 10 \mathrm{keV}$ electrons into the chromosphere at a rate $10^{36} \mathrm{~s}^{-1}$. It is thought that $\gtrsim 20 \%$ of the magnetic energy released during the impulsive phase goes into these electrons (Duijveman, Hoyng, \& Machado 1982). The mechanism for the bulk energization of the electrons is not understood in detail. The deca-MeV ions and relativistic electrons are required to produce the gamma-ray bursts that begin within $\sim 1 \mathrm{~s}$ of the onset of the impulsive phase of some flares. There are several different theories for their acceleration, with stochastic acceleration by MHD turbulence and shock acceleration being two possibilities. In this review, some ideas on turbulent acceleration are applied to the acceleration of these particles in the impulsive phase, with the emphasis on the bulk energization of electrons.

Possible acceleration mechanisms can be separated into four broad classes: acceleration by shock waves, acceleration by parallel electric fields, acceleration in neutral sheets, and turbulent acceleration. These are discussed in $\S 2$. The role of currentdriven low-frequency electrostatic turbulence is discussed in $\S 3$, and the acceleration of electrons in the presence of such turbulence is reviewed in $\S 4$. Acceleration by MHD turbulence is discussed in $\S 5$.

\footnotetext{
${ }^{1}$ E-mail: melrose@physics.su.oz.au
}

\section{ACCELERATION MECHANISMS AND SOURCES OF WAVE TURBULENCE}

The four broad classes of acceleration mechanisms mentioned above are described briefly here, and then possible sources of the wave turbulence required for turbulent acceleration are outlined.

\subsection{Acceleration by Shock Waves}

Acceleration by a (fast-mode) shock wave may separated into two forms: diffusive shock acceleration (DSA) and shock drift acceleration (SDA). DSA requires effective scattering such that a given particle cycles across the shock many times (e.g., the reviews by Drury 1983; Blandford \& Eichler 1987; Jones \& Ellison 1991). DSA is the widely accepted mechanism for the acceleration of Galactic cosmic rays. Although it is a plausible mechanism for the acceleration of higher energy solar energetic particles, the available evidence suggests that prompt acceleration of ions is not due to a single strong shock (Nakajima et al. 1990). DSA by a collection of weak shocks remains a possibility, and this may be treated as a form of acceleration by MHD (fast-mode) turbulence. SDA occurs due to a single transmission or reflection at a shock front causing a particle to gain energy (e.g., the reviews by Toptygin 1980; Pesses, Decker, \& Armstrong 1982; Armstrong, Pesses, \& Decker 1985; Decker 1988). The acceleration may be attributed to an electric field in the shock front, together with the grad $B$ drift being in the direction to cause an energy gain. SDA is important at planetary bow shocks and at interplanetary shocks, but there is no evidence that it is important in the impulsive phase of solar flares.

\subsection{Acceleration by Parallel Electric Fields}

The simplest form of acceleration is when there is an electric field parallel to the ambient magnetic field, $\boldsymbol{B}$, when ions are 
accelerated in one direction and electrons in the other. Plasmas are highly conducting (low electrical resistivity), and parallel electric fields are rapidly shorted out except in localized regions, which can be of several different forms. Examples include regions of anomalous resistivity, due to enhanced levels of low-frequency electrostatic waves, and double layers, which fall into two classes: weak and strong double layers. A weak double layer (WDL) is a specific structure that relies on anomalous resistivity, such that the parallel potential drop forms over a localized region with a turbulent wave spectrum. The potential drop across a WDL is of order $T_{e} / e$, where $T_{e}$ is the electron temperature (in energy units). It is thought that a large potential drop, $\gg T_{e} / e$, imposed along field lines in a plasma, separates into a series of many WDL (e.g., Smith 1982). As discussed below, acceleration by a collection of WDLs may be regarded as turbulent acceleration by low-frequency electrostatic waves. Strong double layers have been discussed extensively in connection with astrophysical plasmas (e.g., Raadu 1989), but are not discussed here.

\subsection{Acceleration in Neutral Sheets}

A neutral sheet separating two regions of oppositely directed magnetic field necessarily contains an electric field. A particle that enters the sheet has a high probability of emerging from it with a higher energy due to a net drift in the direction of decreasing electric potential energy. Acceleration of ions at neutral sheets has been considered in considerable detail recently in connection with current sheets in Earth's magnetotail (e.g., Büchner \& Zelenyi 1989; Burkhart et al. 1992; Chen 1992). Similar acceleration occurs in more complicated geometries, such as that in current loop coalescence (e.g., Sakai 1990). Application of this mechanism to solar flares is not discussed further in this paper.

\subsection{Turbulent Acceleration}

Turbulent acceleration may be defined as acceleration by a spectrum of waves. The acceleration is due to the electric field in the wave, and the energy gain of the particles is at the expense of the waves, which are damped. For turbulent acceleration to be effective three conditions must be satisfied: (1) there must be an adequate energy source for the waves, (2) there must be energy exchange mechanism that allows the particles to gain energy thereby causing the waves to damp, and (3) there must be an adequate supply of particles available to be accelerated. There are two plausible energy sources for waves: large-scale current systems that lead to current instabilities, and mass motions that result in MHD turbulence. In all cases discussed here, the energy exchange involves a resonant interaction between the particles and the waves. For a wave with frequency $\omega$ and wave vector $k$, the resonance condition is

$$
\omega-\boldsymbol{k} \cdot \boldsymbol{v}=0
$$

or

$$
\omega-s \Omega-k_{\|} v_{\|}=0
$$

where equation ( 1a) applies for an unmagnetized particle with velocity $v$ and equation ( $1 \mathrm{~b}$ ) is for resonance at the $s$ th har- monic for a magnetized particle of charge $q$, mass $m$, Lorentz factor $\gamma$, and velocity $v_{\|}$parallel to the magnetic field, where $\Omega=|q| B / m \gamma$ is the gyrofrequency. The ratio of changes in the energy and momentum of the particle interacting with a given wave are in the ratio $\omega$ to $k$, respectively. Retention of the term $\omega$ is essential in equation ( $1 \mathrm{~b}$ ) when discussing acceleration or other energy changes.

\subsection{Current-driven Turbulence}

A current instability involves waves being driven unstable by a current due to the drift speed, $v_{d}$, exceeding a relevant threshold, where the current density is written $J=e n_{e} v_{d}$. Current-driven turbulence can consist of various forms of lowfrequency electrostatic waves, including ion sound waves, Buneman waves, electrostatic ion cyclotron waves, lower hybrid waves, and resonant whistler waves, e.g., as discussed by Duijveman, Hoyng, \& Ionson (1981) and by Holman (1985) in the present context. Some of the effects of such turbulence can often be described in terms of anomalous transport coefficients, which have the same functional form as collision-dominated transport coefficients, with the collision frequency replaced by an effective collision frequency, $\nu_{\text {eff }}$, that depends on the details of the turbulent spectrum.

\subsection{MHD Turbulence}

Generation of MHD turbulence can occur when a macroscopic system supported by magnetic stresses becomes unstable. The generation of MHD turbulence in coronal loops due to wiggling of the footprints by photospheric motions has been discussed extensively in connection with the heating of the corona (e.g., van Ballegooijen 1986; Beaufumé, Coppi, \& Golub 1992). However, as a source for flares, photospheric motions are inadequate from an energetic viewpoint (e.g., McClymont \& Fisher 1989). It is likely that MHD turbulence is generated during magnetic reconnection, but there is no detailed theory for this, and the fraction of the magnetic energy that goes into MHD turbulence is not known. In treating acceleration by MHD turbulence, it is usually simply postulated that the turbulence is present, and the question asked is what level and form of turbulence is required to account for the observed fast particles.

\subsection{Langmuir Turbulence}

A seemingly favorable alternative to current-driven turbulence or MHD turbulence is Langmuir turbulence, which would be very effective in accelerating electrons (e.g., Tsytovich 1966). However, there appears to be no adequate energy source for the Langmuir waves. The most effective source of Langmuir turbulence is a beam instability, as in a type III radio source. However, the Langmuir turbulence remains highly localized within the beam and so can produce only highly localized heating and acceleration. Moreover, an appeal to a beam to generate the Langmuir waves leads to a circular argument from an energetic viewpoint. The objective is to identify the waves that accelerate effectively all the electrons in a localized region and invoking beam-excited Langmuir waves requires that accelerated electrons already be present and supply the energy to the Langmuir waves. One suggested mechanism for 
generating Langmuir waves involves upconversion from ion sound turbulence (Tsytovich, Stenflo, \& Wilhelmsson 1975), but this mechanism has been rejected on both practical (Heyvaerts 1981) and fundamental (Melrose \& Kuijpers 1987) grounds. Although there is evidence for the presence of Langmuir waves in solar flares, through plasma emission at the fundamental and second harmonic of the plasma frequency, the inferred level of Langmuir turbulence is too small, and too localized, for it to be of energetic importance in the bulk acceleration of electrons.

\section{ANOMALOUS RESISTIVITY AND MARGINAL STABILITY}

It is widely accepted that the energy release in the impulsive phase of solar flares, leading to bulk energization of electrons, results from annihilation of magnetic energy or, equivalently, from dissipation of electric currents in the corona. Joule dissipation, due to the collisional (Spitzer) resistivity, cannot account for the power released in a flare. It follows that some enhanced form of dissipation is essential. However, before showing this it is appropriate to remark on the resistivity of a plasma.

\subsection{Anomalous Resistivity}

The power dissipated per unit volume by a current, $J$, is $\eta J^{2}$, where $\eta$ is the resistivity. This follows from Maxwell's equations, which imply a power dissipated $\boldsymbol{J} \cdot \boldsymbol{E}$, together with the phenomenological relation $\boldsymbol{J}=\eta \boldsymbol{E}$. The value of $\eta$ is determined by the effective frictional force between the electrons and the ions. To see this, write the current density in the form $J=e n_{e} v_{d}$, where $v_{d}$ is the drift speed of the electrons relative to the ions. The electric field, $E$, which is attributed to the changing magnetic field in the present case, tends to increase $v_{d}$, and the friction causes $v_{d}$ to decrease at a rate $\nu_{\text {eff }} v_{d}$, where $\nu_{\text {eff }}$ is the effective collision frequency. A balance between these two effects implies $\boldsymbol{J}=\eta \boldsymbol{E}$ with

$$
\eta=\nu_{\mathrm{eff}} \frac{\epsilon_{0}^{2}}{\omega_{p}} \approx\left\{\begin{array}{c}
1.3 \times 10^{3} T_{e}^{-3 / 2} \Omega \mathrm{m} \\
\quad \text { (collisional) } \\
0.2\left(\frac{\xi}{10^{-2}}\right)\left(\frac{n_{e}}{10^{16} \mathrm{~m}^{-3}}\right)^{-1 / 2} \Omega \mathrm{m} \\
\text { (anomalous) }
\end{array}\right.
$$

where $T_{e}$ is the electron temperature in kelvins, and with $\xi=$ $\nu_{\text {eff }} / \omega_{p}$. In the collisional case, $\nu_{\text {eff }}$ is due to Coulomb interactions. Anomalous resistivity occurs when the drift velocity, $v_{d}$, exceeds an appropriate threshold causing appropriate waves to grow. The waves carry off some of the directed momentum of the electrons, providing a friction-like drag on the electrons that is much more effective than that due to Coulomb interactions. The resistivity is then said to be anomalous, as discussed in the present context by, e.g., Duijveman et al. (1981) and Holman (1985). The value of $\xi$ in equation (2) depends on the details of the turbulence (e.g., Papadopoulos 1985), and a value $\xi \sim 10^{-2}$ is considered plausible. Anomalous resistivity leads to the release of magnetic energy into particle energy through the waves. In a steady state, the following processes are involved: (1) the waves grow due to the current instability, (2) the wave energy is transferred from the regions of $\boldsymbol{k}$-space where the waves are growing to other regions of $\boldsymbol{k}$-space through nonlinear processes in the plasma, (3) in these other regions of $\boldsymbol{k}$-space the waves are damped, heating the electrons.

Although a variety of wave modes needs to be considered in a general treatment of anomalous resistivity, in this paper only ion sound waves are considered. Ion sound waves have a dispersion relation $\omega \approx k v_{s}$ for $k \ll \lambda_{\mathrm{D}}$, where $v_{s}=V_{e} / 43$ is the ion sound speed and $\lambda_{\mathrm{D}}=V_{e} / \omega_{p}$ is the electron Debye length. The threshold for the onset of the instability is $v_{d}>v_{s}$. This may be seen by noting that the instability occurs approximately for $\omega-\boldsymbol{k} \cdot \boldsymbol{v}_{d}=0$, which with $\omega=k v_{s}$ implies $v_{d}>v_{s}$. Ion sound waves exist only for $T_{e} \gg T_{i}$, and other forms of wave turbulence can cause anomalous resistivity when the condition $T_{e} \gg$ $T_{i}$ is not satisfied. Suppose $T_{e} / T_{i}$ were too small for ion sound waves to exist, so that other low-frequency electrostatic turbulence would be generated by a current instability. These waves would damp preferentially heating the electrons, tending to increase $T_{e} / T_{i}$ until the ion sound instability takes over. Hence, although the primary reason here for concentrating on ion sound waves is simplicity, the foregoing argument suggests that these waves are plausibly the most important in practice.

\subsection{The Need for Anomalous Resistivity}

The argument that the collisional (Spitzer) resistivity is inadequate and that anomalous resistivity is essential in the dissipation in solar flares can be summarized as follows (e.g., Chiuderi 1981, 1983; Martens, van den Oord, \& Hoyng 1985; Melrose \& McClymont 1987). Let the current flow in a channel of cross-sectional area $A$, so that the total current is $I=J A$. There is a maximum value of the current for the flux tube not to be unstable to kinking. For a cylindrical current $\left(A=\pi r^{2}\right)$ this is $I<2 \pi r B / \mu_{0}$, where $B$ is the axial magnetic field. In a solar flare the observed currents, $\lesssim 10^{13} \mathrm{~A}$, that correlate with flare kernels (e.g., Lin \& Gaizauskas 1987; Machado et al. 1988 ) are close to this limit. The power dissipated, which is identified here as the power released in the flare, may be estimated by multiplying $\eta J^{2}$ by the volume $V=A l$ of a length $l$ of the current element. It then follows that for fixed $\eta, I$, and $l$ the power dissipated is maximized by minimizing the cross-sectional area $A$, which is equivalent to maximizing the current density $J=I / A$ and hence $v_{d}$. For anomalous resistivity to be unimportant $v_{d}$ must remain below the threshold for the current instability. Assuming $J=e n_{e} v_{s}$, this gives a maximum power that can be dissipated due to the Spitzer resistivity, which for plausible numbers for a solar flare reduces to

$$
\begin{array}{r}
P_{\max }=\eta I J l=2 \times 10^{15}\left(\frac{T_{e}}{10^{7} \mathrm{~K}}\right)^{-1}\left(\frac{l}{10^{4} \mathrm{~km}}\right) \\
\times\left(\frac{n_{e}}{10^{16} \mathrm{~m}^{-3}}\right)\left(\frac{I}{10^{13} \mathrm{~A}}\right) \mathrm{W}
\end{array}
$$

The observed power in a moderate flare is $\sim 10^{21} \mathrm{~W}$, and so clearly the Spitzer resistivity is inadequate by many orders of magnitude.

\subsection{Filling Factors}

When dissipation occurs through anomalous resistivity the current density is necessarily above threshold. A filling factor 
for the cross-sectional area in which the current flows may be estimated by comparing the effective area $I / e n_{e} v_{s}$ of the current channels with the total area of the flux tube in which the current flows. The filling factor for the current is $\sim 10^{-4}-$ $10^{-3}$. The wave turbulence must also be localized along the direction of current flow. This may be seen by noting that the electric field at marginal stability is determined as $E=\eta e n_{e} v_{s}$, with $\eta$ given by the collisionless case in equation (2). On multiplying this value of $E$ by the net length of the current path that is anomalously resistive, the total potential drop should not exceed the maximum available $\sim 10^{10} \mathrm{~V}$. Using such arguments a very small, but highly uncertain total filling factor is implied (Melrose 1990). The filling factor of the region in which heating is occurring at any given time is estimated below and appears to be considerably larger than the very small value suggested by the foregoing argument.

The localization of the potential drop across many anomalously resistive regions is similar to what is expected in multiple WDLs (e.g., Smith 1982). The acceleration of electrons in the auroral zones of Earth is thought to involve a collection of WDLs (e.g., Boström et al. 1989), although there is a contrary view (e.g., Bryant 1990). Also, the observed structure of the WDLs (cf. Fig. 1) does not appear to be consistent with that expected for anomalous resistivity, and a model based on ion phase space holes may be more appropriate (e.g., Mälkki et al. 1989; Tetreault 1991). The possible relevance of such ideas in the solar context has been discussed elsewhere (Melrose 1992).

\subsection{Marginal Stability Analysis and Self-organized Criticality}

The plasma processes related to the current instability and the wave turbulence involve lengths of at most hundreds to thousands of Debye lengths. From the viewpoint of the macroscopic dimensions involved in the overall energy release, such length scales are microscopic. The two scales differ by a factor of at least $10^{5}$. It is physically implausible that the details of the localized microscopic regions of wave turbulence can be important in determining the evolution of the macroscopic system. Individual localized regions may have a rapidly changing structure, but plausibly the ensemble varies only slowly in both space and time as the macroscopic structure evolves. One
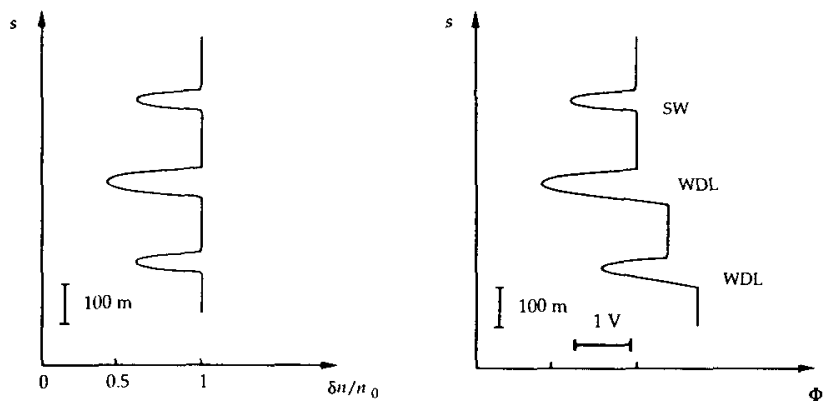

FIG. 1.-Variation of the relative density $\left(\delta n / n_{0}\right)$ and potential $(\Phi)$ with height $s$ observed above the auroral zones from the Viking spacecraft; SW denotes a solitary wave, and WDL denotes a weak double layer. (After Böstrom et al. [1989] and Mälkki et al. [1989].) needs a specific model for such an ensemble in order to link the microscopic and macroscopic systems, and this requires some additional assumption.

One pausible way to proceed is to appeal to marginal instability, sometimes called marginal stability analysis (e.g., Manheimer \& Lashmore-Davies 1989). The basic idea may be described as follows in the present context. Once the instability develops, its backreaction tends to reduce the drift velocity until it is close to the threshold value. Over times long compared to the growth rate of the instability and short compared to the time scale for changes of the macroscopic system, one expects the drift velocity to be maintained at close to the threshold value. It is argued that near marginal stability the growth rate (averaged over space and time) adjusts to produce a rate of dissipation or other evolution determined by macroscopic boundary conditions. Marginal stability analysis was applied to anomalous resistivity in the solar corona by Duijveman et al. (1981). A similar idea was referred to as stochastic-growth theory by Robinson (1993), who applied it to the beam instability in type III radio bursts. A related type of argument leads to the concept of self-organized criticality, introduced by Bak, Tang, \& Wiesenfeld ( 1987) and applied to solar flares by Lu \& Hamilton (1991). It is argued that the structure of unstable systems becomes scale independent, on a macroscopic scale, as a result of the instabilities being maintained near threshold. The important implications of these ideas is that they provide a link between the microphysics, as in the details of the anomalous resistivity, and the global structure and evolution of the system.

\section{BULK ENERGIZATION AND RUNAWAY ACCELERATION}

It is assumed here that the primary energy release in a flare results in most of the energy going initially into bulk energization of electrons. It is then required that effectively all the electrons in the flaring region have their mean energy increased manyfold during the flare. This bulk energization cannot be due to collisional effects, as shown above, and is due to the damping of the wave turbulence.

\subsection{Bulk Heating Due to Low-Frequency Turbulence}

Damping of waves is a very efficient heating mechanism provided an adequate source of the wave energy is present. Consider ion sound turbulence with an energy density $W_{s}$ and a mean wavenumber $\bar{k}$. The local heating rate for the thermal electrons, $n_{e} \dot{T}_{e}$, may be estimated from the rate the wave energy damps,

$$
n_{e} \dot{T}_{e}=\gamma_{s} W_{s}, \quad \gamma_{s} \sim \bar{k} v_{s}^{2} / V_{e}
$$

However, the heating rate is already identified in the model as the rate the current dissipates, which implies $n_{e} \dot{T}_{e}=\eta J^{2}$. The latter is to be evaluated with $\eta$ given by equation (2) and with $J \sim n_{e} e v_{s}$ near the threshold for instability. Equating the two estimates of the heating rate implies a value for $\xi$ in equation (2): $\xi \sim\left(\dot{k} \lambda_{\mathrm{D}}\right)\left(W_{s} / n_{e} T_{e}\right)$. For the sake of discussion, suppose one has $\xi \sim 10^{-2}$ due to $W_{s} / n_{e} T_{e} \sim 10^{-2}$ for $\bar{k} \lambda_{\mathrm{D}} \sim 1$. The implied local heating rate gives an exponential increase in the electron temperature with an $e$-folding rate $\gamma_{s}\left(W_{s} / n_{e} T_{e}\right)$. For 
parameters chosen here this rate is very high, $\sim 3 \times 10^{4} \mathrm{~s}^{-1}$. In fact this is an unacceptably high rate because it would imply that the ion sound turbulence is removed faster than it is generated. This discrepancy may be resolved by assuming that, at any given time, the volume in which the wave energy is dissipated, and in which the bulk energization occurs, is much larger than the volume in which the waves are generated.

The volume to which the plasma heating is confined may be estimated by considering the ratio of the power released in a flare, say $10^{21} \mathrm{~W}$, and the power per unit volume, $\gamma_{s} W_{s}$, transferred in the heating process. This gives a filling factor $\sim 10^{4}\left(n_{e} T_{e} / W_{s}\right) / \gamma_{s} t_{f}$, where $t_{f}$ is the life time of the flare. This is much larger than the filling factor estimated above for the regions in which the waves are being generated, and is of order the factor $10^{-4}$ estimated from observation for a flux tube being heated by this mechanism (Martens et al. 1985).

The requirement that the wave energy be dissipated in a much larger volume than that in which it is generated can be satisfied only if the damping is considerably slower than the growth and only if propagation effects allow the turbulence to spread out. The relative slowness of the dissipation may be understood as follows. As noted above, the generation of the waves occurs in one region of $\boldsymbol{k}$-space and they need to be transferred, by nonlinear processes in the plasma, to other regions of $\boldsymbol{k}$-space where they damp. The rate of damping of the wave energy is limited by the relatively slow rate at which this transfer occurs in the marginally stable state. The spreading out of the turbulence could be due to either the propagation of waves away from their sources (the current channels) or to motion of the current channels. The latter of these should be the more important: the current channels tend to avoid regions of high resistivity, and once anomalous resistivity develops locally the current channel moves away into a neighboring region of lower resistivity, causing the waves to grow in this neighboring region. This leads to a picture in which the wave turbulence is left in a wake as the current channels are driven across the field lines. How this motion of current channels occurs involves the transfer of stresses through Alfvén waves (e.g., Haerendel 1987; Melrose 1992).

\subsection{Runaway Acceleration}

Acceleration of particles by waves generated through a current instability occurs in the presence of the electric field that drives the current. The effect of both the waves and the DC electric should be taken into account simultaneously. A plausible model for the acceleration of electrons to higher energy, to form a nonthermal tail on the hot electron distribution, is runaway acceleration by the electric field (e.g., Kuijpers, van der Post, \& Slottje 1981; Holman 1985; Takakura 1987, 1988; Holman, Kundu, \& Kane 1989). Runaway acceleration results from a DC electric field, $E$, along the ambient magnetic field. The acceleration $-e E / m_{e}$ of electrons is opposed by a collision-like drag provided by the ion sound turbulence. For electrons with speed much greater than the phase speed of the waves, the drag has the same functional form as for Coulomb interactions: $\nu_{\text {eff }}(v) \sim \nu_{\text {eff }}\left(v / V_{e}\right)^{-3}$, with $\nu_{\text {eff }}$ given by equation (2). The usual Dreicer field is replaced by an effective Dreicer field by replacing the Coulomb collision frequency by the effective collision frequency:

$$
E_{\mathrm{D}}=\frac{m_{e} V_{e}^{+}}{e} \nu_{\mathrm{eff}}
$$

The threshold for runaway acceleration is $v_{c}>\left(E / E_{\mathrm{D}}\right)^{1 / 2} V_{e}$, that is, all electrons with $v>v_{c}$ initially are freely accelerated. The rate at which such runaways occur is a sensitive function of $v_{c} / V_{e}$ (e.g., Holman 1985), and hence is a sensitive function of $W_{s} / n_{e} T_{e}$ through the dependence of $\nu_{\text {eff }}$ and hence of $E_{\mathrm{D}}$ on this parameter.

The rate at which runaway electrons are produced is limited by the requirement that the total current not change significantly (Cowling 1953; Spicer 1983; Holman 1985). Hence the rate cannot exceed $I / e$, which is $\sim 10^{34} \mathrm{~s}^{-1}$ for $I \sim 10^{13} \mathrm{~A}$. In a marginally stable state the average rate of runaways presumably adjusts so that the current carried by the runaway electrons carry does not violate this constraint.

\subsection{Runaway Acceleration in Ion Sound Turbulence}

Takakura $(1987,1988)$ developed a model to treat some of the effects of ion sound turbulence described above. The electric field was attributed to the decay of a force-free magnetic field and the resistivity was assumed of the form given by equation (2) with $\xi \sim 10^{-2}-10^{-3}$. For one localized region with potential drop $\Phi$, Takakura ( 1988) found that the spectrum of runaway electrons is hard, with a power-law-like spectrum with a spectral index $\delta \sim 0.75$ up to a maximum $e \Phi$. In order to account for the observed hard X-ray spectra from solar flares, Takakura (1988) assumed that there are very many localized regions of acceleration, each with a different $\Phi$. Assuming a power-law ensemble distribution of these regions in $\Phi$, a convolution the spectrum for a given $\Phi$ with the distribution of values of $\Phi$ was shown capable of accounting for the observed spectra.

\section{ACCELERATION BY MHD TURBULENCE}

Acceleration by MHD turbulence can occur through two main types of interaction: stochastic acceleration and gyroresonant acceleration. Both types of acceleration are possible only when a threshold condition on the particle energy is exceeded, so that these acceleration mechanisms require preacceleration to above the relevant threshold.

\subsection{Stochastic Acceleration}

The concept of stochastic acceleration developed from the older ideas of (second-order) Fermi acceleration, magnetic pumping, and transit acceleration by MHD turbulence. The nature of stochastic acceleration was clarified by Tverskoi (1967), who showed that it may be described by isotropic diffusion in momentum space, by Kulsrud \& Ferrari (1971), who emphasized the role of resonant scattering and showed how the three different energy exchange mechanisms are related, and by Achterberg (1981a), who showed that the energy exchange corresponds to Landau damping of MHD waves, corresponding to resonance at $s=0$ in equation ( $1 \mathrm{~b}$ ). The resonant interaction at $s=0$ is ineffective for Alfvén waves, and causes damping only of the compressional component (fast mode) in the MHD turbulence. The effect of the interaction at $s=0$ on the particles is to change the parallel momentum component, 
$p_{\|}$, leaving $p_{\perp}$ unaffected. As shown by Achterberg (1981a), the resulting diffusion in $p_{\|}$is self-limiting; resonant scattering is required to prevent the acceleration suppressing itself.

Assuming that resonant scattering is effective in maintaining the distribution close to isotropy, the diffusion in momentum space for the isotropic part has the form

$$
\frac{\partial}{\partial t}\langle f\rangle(p)=\frac{1}{p^{2}} \frac{\partial}{\partial p}\left[p^{2} D_{p p}(p) \frac{\partial}{\partial p}\right]\langle f\rangle(p),
$$

where $\langle f\rangle(p)$ is the particle distribution function averaged over pitch angle, and with

$$
D_{p p}(p)=\nu_{A} \frac{c p^{2}}{4 v}\left(1-\frac{v_{\mathrm{A}}^{2}}{v^{2}}\right)^{2}, \quad \nu_{A}=\frac{\pi}{4} \bar{\omega}\left(\frac{\delta B}{B}\right)^{2},
$$

where $\nu_{A}$ is the acceleration rate with $\delta B$ the magnetic amplitude in the waves and $\bar{\omega}$ their mean frequency. A remarkable feature of equation ( 7 ) is that the acceleration rate is independent of the details of the scattering of the particles, and yet effective scattering is an essential ingredient in the theory, cf. Kulsrud \& Ferrari (1971) and Achterberg (1981a).

In principle, stochastic acceleration can be very effective and quite rapid, but there are two aspects of it that raise doubts about its effectiveness in solar flares: the level of the MHD turbulence, and the injection of fast particles. With $\delta B \sim B$ in equation ( 7 ), the rate of acceleration can be fast enough to account for the prompt acceleration of the energetic ions that produce gamma rays in solar flares (e.g., Melrose 1983; Miller \& Ramaty 1987; Smith \& Brecht 1989; Steinacker, Dröge, \& Schlickeiser 1989; Schlickeiser \& Steinacker 1989; Miller $1991)$. In addition, when source and loss terms are included in equation (6), the solution has a Bessel-function spectral form for which there is observational support (e.g., Forman, Ramaty, \& Zweibel 1986). However, there is no direct information on the level of MHD turbulence in a flaring region, and one can only conclude that if stochastic acceleration is operating then the promptness implies that the turbulence is maintained at the required level. A more quantitative discussion of this point was given by Miller, Guessoum, \& Ramaty (1990).

DSA by an ensemble of weak shocks has the same form as stochastic acceleration (e.g., Achterberg 1990). Thus, an ensemble of weak shocks may be regarded, from the point of view of particle acceleration, as equivalent to a distribution of fastmode turbulence. It then follows that when considering the prompt acceleration of ions and relativistic electrons it is not important to distinguish between MHD turbulence in the form of a distribution of small-amplitude waves and an equivalent energy density of in weak shock waves.

\subsection{Role of Alfvén Waves}

In general, MHD turbulence consists of a mixture of two modes, both of which propagate at approximately the Alfvén speed $v_{\mathrm{A}}$ in a low-beta plasma. (There is a third mode, the slow mode, which is not relevant to the discussion here.) Only the fast mode contributes to stochastic acceleration. This raises the question as to whether the Alfvénic component can contribute indirectly to stochastic acceleration. One possibility occurs if the MHD turbulence involves a magnetic cascade (e.g., Bicknell \& Melrose 1982; van Ballegooijen 1986). In a magnetic cascade three ranges are identified: long wavelengths were the waves are generated, an inertial range where the spectrum is determined by the cascade, and a sink at short wavelength where dissipation dominates. The formation of the resulting turbulent spectrum has been discussed recently in connection with the solar wind (e.g., Zhou \& Mattheus 1990). It is possible for stochastic acceleration to be the sink mechanism for the cascade, so that effectively all the turbulent energy ends up in fast particles. According to equation (7), the acceleration becomes more effective as the wavelength $(\propto 1 / \bar{\omega})$ decreases, and the sink occurs at the wavelength (or frequency) at which the rate of dissipation of the turbulence due to the acceleration of fast particles exceeds the rate at which the turbulence cascades to shorter wavelengths. Only the fast-mode component is removed at the wavelength of the sink. However, within the dissipation region the cascade can continue starting with the remaining Alfvénic component, which produces a mixture of Alfvén and fast-mode component at a shorter wavelength, and the resulting fast-mode component is damped rapidly. Hence the Alfvénic component is progressively removed at each subsequent step in the cascade, allowing effectively all the turbulent energy to be transferred to the fast particles through stochastic acceleration.

\subsection{Gyroresonant Acceleration}

An alternative to stochastic acceleration is gyroresonant acceleration, due to interactions at harmonics $|s| \geq 1$ in equation ( $1 b)$. Lacombe (1977) discussed interactions at $|s| \gg 1$ in connection with the acceleration of relativistic particles in extragalactic sources, Barbosa (1979) discussed acceleration at $|s|=1$ in connection with the acceleration of solar protons, and Miller \& Ramaty (1989) applied the mechanism to the acceleration of relativistic electrons. The acceleration in the presence of efficient scattering may be described by an equation of the form $(6)$, with the functional form of the diffusion coefficient $D_{\mathrm{pp}}(p)$ related to the $k$-dependence of the turbulent spectrum, cf. Miller \& Ramaty (1989). Miller et al. (1990) compared gyroresonant acceleration by Alfvén waves and stochastic acceleration by fast-mode waves under conditions thought appropriate to the solar corona, and found the two to be comparable for an energy in Alfvén waves $\sim 5$ times the energy density in fast-mode waves. This suggests that gyroresonant acceleration may be neglected for semiquantitative estimates of the acceleration of ions and relativistic electrons, but that it should be included in any thorough analysis.

\subsection{Threshold Conditions}

The threshold for stochastic acceleration to be effective arises from two separate effects. First, equation ( $1 \mathrm{~b}$ ) with $s=0$, $\omega=k v_{\mathrm{A}}$ for fast-mode waves and $k_{\|}=k \cos \theta$, requires $v_{\|}=$ $v_{\mathrm{A}} / \cos \theta$. This condition can be satisfied only for $v>v_{\mathrm{A}}$, implying that the mechanism operates only for particles (of any species) with speed greater than the Alfvén speed. Second, an effective scattering mechanism is required, and the only plausible candidate is resonant scattering. For ions, resonant scattering involves a spectrum of Alfvén and fast-mode waves ( separate from and usually with a much higher frequency than the MHD turbulence) that interact with the particles at $s= \pm 1$ in equation ( $1 \mathrm{~b})$. The resonance ( $1 \mathrm{~b}$ ) for nonrelativistic ions at $s= \pm 1$ requires $\left|v_{1}\right| \approx\left(\Omega_{p} / \omega\right) v_{\mathrm{A}}$ for Alfvén waves and $\left|v_{\|}\right| \approx$ 
$\left(\Omega_{p} / \omega\right)\left(v_{\mathrm{A}} /|\cos \theta|\right)$ for fast-mode waves. Thus resonant scattering of ions also requires $v>v_{\mathrm{A}}$, cf. Achterberg (1981b) for a more detailed discussion. For nonrelativistic electrons the resonant scattering requires higher frequency waves, usually assumed to be whistler waves, and the threshold speed for resonant scattering is correspondingly higher, $v>43 v_{\mathrm{A}}$. The threshold conditions for gyroresonant acceleration to occur are the same as for resonant scattering. Thus preacceleration mechanisms are required to supply ions and electrons above these respective thresholds for stochastic acceleration or gyroresonant acceleration to operate.

\subsection{Preacceleration Mechanisms}

A suitable preacceleration mechanism must provide a seed population of ions at $v>v_{\mathrm{A}}$ (e.g., the review by Melrose 1990). A constraint on any preacceleration mechanism for ions is provided by data on the relative isotopic abundances in accelerated and thermal ions. For the ions accelerated in the impulsive stage of flares there are specific abundance anomalies (e.g., Reames 1990 ), notably the highly variably ${ }^{3} \mathrm{He} /{ }^{4} \mathrm{He}$ ratio, that need to be explained. Stochastic acceleration is relatively insensitive to the ionic species, and any difference in the relative abundances must be due predominantly to the preacceleration mechanism. The abundance anomalies in impulsive flares, where species-sensitive preacceleration is required, contrast with the abundances of shock-acceleration ions in the solar wind, which reflect the abundances of the ambient thermal ions. Thus, observationally, it seems that a shock can accelerate ions from the thermal distribution and that a preacceleration mechanism is not required for DSA (cf. Galeev, Sagdeev, $\&$ Shapiro 1986). A mechanism involving reflection of the ions from the shock followed by DSA of these ions seems favorable (e.g., Scholer 1993).

The preacceleration of electrons may be due to runaway acceleration that produces the nonthermal electron tail, but it seems that the preacceleration of ions requires a different mechanism from those discussed above. One possible mechanism is nonlinear Landau damping of MHD turbulence (Miller 1991). This damping involves a resonance of the form (1b) with $\omega$ and $k_{\|}$for one wave replaced by the corresponding quantities for a beat between two waves. Miller (1991) found that nonlinear Landau damping can cause acceleration of ions from thermal speeds, and he concluded that the heating and acceleration of protons, due to a combination of these effects, can be the dominant dissipation mechanism for the Alfvén component in MHD turbulence. However, this mechanism was considered in detail only for parallel-propagating waves in a hydrogen plasma, and its effects on the isotopic abundances was not explored. A alternative mechanism that seems more favorable involves electromagnetic hydrogen cyclotron waves generated by streaming electrons which appear capable of accounting for the observed anomalies (Temerin \& Roth 1992; Miller 1993).

\section{Discussion and Conclusions}

The emphasis in this paper is on two forms of turbulent acceleration in solar flares: of nonrelativistic electrons by low frequency electrostatic turbulence, and of nonrelativistic ions and relativistic electrons by MHD turbulence. There is no direct observational evidence on the level of turbulence in either case, and so one can only derive conditions that need to be satisfied in order for the acceleration to be effective. The main points made are the following.

1. Collisional processes cannot account quantitatively for the energy release in the impulsive phase of a solar flare, and some form of anomalous resistivity is needed. The current density must be above an appropriate threshold to generate the wave turbulence through a current-driven instability, implying that the current is confined to narrow filaments.

2. One needs to appeal to a marginal stability assumption or similar assumption to relate the plasma microturbulence processes to the evolution of the macrostructure. The microturbulence processes involve localized regions of high current density, electric fields and wave energy density, and the macroscopic evolution involves release of magnetic energy and heat transport on a large scale.

3. The bulk heating is due to the damping of the wave turbulence. To account quantitatively for the total power released poses significant constraints on any model. In particular the volume filled, at any given time, by the wave turbulence heating the electrons must be much larger than the volume filled by the current filaments that are generating the wave turbulence.

4. Higher energy electrons, forming a nonthermal tail, can be explained in terms of runaway acceleration. However, to reproduce the electron spectrum inferred from observation requires that the runaways occur in an ensemble of regions with a distribution of potential drops that determine the average spectrum (Takakura 1988). It is suggested that the low-frequency turbulence may ensure that the rate of runaway acceleration does not lead to violation of the current restriction (that the total current is unchanged on relevant time scales).

5. Acceleration by MHD turbulence includes stochastic acceleration by fast-mode wave and gyroresonant acceleration by Alfvén waves, which is marginally less efficient than stochastic acceleration. Diffusive shock acceleration by an ensemble of weak shocks may be treated as a form of stochastic acceleration. Stochastic acceleration by MHD turbulence seems capable of accounting for the prompt acceleration of ions and relativistic electrons in solar flares.

6. A preacceleration mechanism is required for stochastic acceleration by MHD turbulence to provide a seed population of particles above threshold $\left(v>v_{\mathrm{A}}\right.$ for ions and $v>43 v_{\mathrm{A}}$ for electrons). No adequate preacceleration mechanism has been identified for electrons. For ions a mechanism (Temerin \& Roth 1992) that can account for the observed anomalous abundances seems favorable.

\section{REFERENCES}

Achterberg, A. 1981a, A\&A 97, 259

. 1981b, A\&A, 98, 161

1990, A\&A, 231, 251

Armstrong, T. P., Pesses, M. E., \& Decker, R. B. 1985, in Collisionless

Shocks in the Heliosphere: Reviews of Current Research, ed. B. T. Tsurutani \& R. G. Stone (Washington, DC: AGU), 271

Bak, P., Tang, C., \& Wiesenfeld, K. 1987, Phys. Rev. Lett., 59, 381
Barbosa, D. D. 1979, ApJ, 233, 383

Beaufumé, P., Coppi, B., \& Golub, L. 1992, ApJ, 393, 396

Bicknell, G. V., \& Melrose, D. B. 1982, ApJ, 262, 511

Blandford, R. D., \& Eichler, D. 1987, Phys. Rep., 154, 1

Boström, R., Holback, B., Holmgren, G., \& Koskinen, H. 1989, Phys. Scripta, 33, 523

Bryant, D. A. 1990, Phys. Scripta, T30, 215 
Büchner, J., \& Zelenyi, L. M. 1989, J. Geophys. Res., 94, 11,821

Burkhart, G. R., Drake, J. F., Dusenbery, P. B., \& Speiser, T. W. 1992, J. Geophys. Res., 97, 13,799

Chen, J. 1992, J. Geophys. Res., 97, 15,011

Chiuderi, C. 1981, in Solar Phenomena in Stars and Stellar Systems, ed. R. M. Bonnet \& A. K. Dupree (Dordrecht: Reidel), 269

. 1983, in Solar and Stellar Magnetic Fields: Origins and Coronal Effects, ed. J. O. Stenflo (Dordrecht: Reidel), 375

Cowling, T. G. 1953, in The Sun, ed. G. P. Kuiper (Univ. of Chicago Press), 532

Decker, R. B. 1988, Space Sci. Rev., 48, 195

Drury, L. O'C. 1983, Rep. Prog. Phys., 46, 973

Duijveman, A., Hoyng, P., \& Ionson, J. A. 1981, ApJ, 245, 721

Duijveman, A., Hoyng, P., \& Machado, M. E. 1982, Sol. Phys., 81, 137

Forman, M. A., Ramaty, R., \& Zweibel, E. G. 1985, in The Physics of the Sun, Volume II, ed. P. A. Sturrock, T. E. Holtzer, D. M. Mihalas, \& R. K. Ulrich (Dordrecht: Reidel), 249

Galeev, A. A., Sagdeev, R. Z., \& Shapiro, V. D. 1986, in Proc. Joint Varenna-Abastumani International School and Workshop Plasma Astrophysics (ESA: SP-251), 297

Haerendel, G. 1987, in Proc. 21st ESLAB Symp. (ESA SP-275), 205

Heyvaerts, J. 1981, in Solar Flare Magnetohydrodynamics, ed. E. R. Priest (New York: Gordon \& Breach), 429

Holman, G. D. 1985, ApJ, 293, 584

Holman, G. D., Kundu, M. R., \& Kane, S. R. 1989, ApJ, 345, 1050

Hudson, H. S. 1985, Sol. Phys., 100, 515

Jones, F. C., \& Ellison, D. C. 1991, Space Sci. Rev., 58, 259

Kuijpers, J., van der Post, P., \& Slottje, K. 1981, A\&A, 103, 331

Kulsrud, R. M., \& Ferrari, A. 1971, Ap\&SS, 12, 302

Lacombe, C. 1977, A\&A, 54, 1

Lin, Y., \& Gaizauskas, V. 1987, Sol. Phys., 109, 81

Lu, E. T., \& Hamilton, R. J. 1991, ApJ, 380, L89

Machado, M. E., Moore, R. L., Hernandez, A. M., Rovira, M. G., Hagyard, M. J., \& Smith, J. B., Jr. 1988, ApJ, 326, 425

Mälkki, A., Koskinen, H., Boström, R., \& Holback, B. 1989, Phys. Scripta, 39,787

Manheimer, W. M., \& Lashmore-Davies, C. N. 1989, MHD and Microinstabilities in Confined Plasma (Bristol: Adam Hilger), 241

Martens, P. C. H., van den Oord, G. H. J., \& Hoyng, P. 1985, Sol. Phys., 96,253

McClymont, A. N., \& Fisher, G. H. 1989, in Solar System Plasma Physics, ed. J. H. Waite, J. L. Burch, \& R. L. Moore (Washington, DC: AGU), 219
Melrose, D. B. 1983, Sol. Phys., 89, 149

1990, Australian J. Phys., 43, 703

1992, in Eruptive Solar Flares, ed. Z. Şvestka, B. V. Jackson, \&

M. E. Machado (Berlin: Springer), 147

Melrose, D. B., \& Kuijpers, J. 1987, ApJ, 323, 338

Melrose, D. B., \& McClymont, A. N. 1987, Sol. Phys., 113, 241

Miller, J. A. 1991, ApJ, 376, 342 1993, ApJS, 90,833

Miller, J. A., Guessoum, N., \& Ramaty, R. 1990, ApJ, 361, 701

Miller, J. A., \& Ramaty, R. 1987, Sol. Phys., 113, 195 1989, ApJ, 344, 973

Nakajima, H., Kawashima, S., Shinohara, N., Shiomi, Y., \& Enome, S. 1990, ApJS, 73, 177

Papadopoulos, K. 1985, in Collisionless Shocks in the Heliosphere: A Tutorial Review, ed. R. G. Stone \& B. T. Tsurutani (Washington, DC: AGU), 59

Pesses, M. E., Decker, R. B., \& Armstrong, T. P. 1982, Space Sci. Rev., 32, 185

Raadu, M. A. 1989, Phys. Rep., 178, 27

Ramaty, R., et al. 1980, in Solar Flares, ed. P. A. Sturrock (Boulder: Colorado Univ. Press), 117

Reames, D. V. 1990, ApJS, 73, 235

Robinson, P. A. 1993, Sol. Phys., 146, 357

Sakai, J.-I. 1990, ApJS, 73, 321

Schlickeiser, R., \& Steinacker, J. 1989, Sol. Phys., 122, 29

Scholer, M. 1993, JGR, 98, 47

Smith, D. F., \& Brecht, S. H. 1989, ApJ, 344, 1004

Smith, R. A. 1982, Phys. Scripta, T2, 238

Spicer, D. S. 1983, Adv. Space Res., 2, 135

Steinacker, J., Dröge, W., \& Schlickeiser, R. 1989, Sol. Phys., 115, 313

Takakura, T. 1987, Sol. Phys., 107, 283

- 1988, Sol. Phys., 115, 149

Temerin, M., \& Roth, I. 1992, ApJ, 391, L105

Tetreault, D. 1991, J. Geophys. Res., 96, 3549

Toptygin, I. N. 1980, Space Sci. Rev., 26, 157

Tsytovich, V. N. 1966, Soviet Phys.-Uspekhi, 9, 370

Tsytovich, V. N., Stenflo, L., \& Wilhelmsson, H. 1975, Phys. Scripta, 11, 251

Tverskol, B. A. 1967, Soviet Phys._JETP Lett., 25, 317

van Ballegooijen, A. 1986, ApJ, 311, 1001

Zhou, Y., \& Mattheus, W. H. 1990, J. Geophys. Res., 95, 10,291 